Running head: RELIABILITY OF VINELAND-3

\title{
Reliability of the Vineland Adaptive Behavior Scales, Third Edition
}

Cristan Farmer ${ }^{1}$, Dee Adedipe ${ }^{1}$, Vanessa Bal ${ }^{2}$, Colby Chlebowski ${ }^{1}$, Audrey Thurm ${ }^{1}$

${ }^{1}$ Neurodevelopmental and Behavioral Phenotyping Service, Intramural Research Program, National Institute of Mental Health

${ }^{2}$ Graduate School of Applied and Professional Psychology, Rutgers University

Correspondence: Cristan Farmer, 10 Center Drive, Bethesda MD 20892. Ph: (301) 435-3999

Acknowledgments: This work was supported in part by the Intramural Research Program of the National Institute of Mental Health (1ZICMH002961; NCT00271622) and K23MH115166 to VB. We thank Megan Fok and Alexis Sullivan for their assistance in data collection.

Conflicts of interest: CF, DA, VB, CC, and AT report no real or potential conflicts of interest. 


\begin{abstract}
Background: Due to its centrality in the conceptualization of intellectual disability, reliable and valid measurement of adaptive behavior is important to both research and clinical practice. The manual of the Vineland Adaptive Behavior Scales, recently released in its third edition, provides limited reliability information obtained from a sample composed primarily of typically developing individuals. The goal of this study was to evaluate the reliability of the Vineland-3 with the Vineland-II in a sample more similar in ability level to those in which the Vineland is commonly used.
\end{abstract}

Methods: Both editions of the Vineland Comprehensive Parent Interviews were conducted with a convenience sample of 106 parents/caregivers of individuals with neurodevelopmental disability, participating at two neurodevelopmental disorder research clinics. Administrations were up to 7 days apart, but most (90\%) were simultaneous. The concordance correlation coefficient (CCC) $[95 \% \mathrm{CI}]$ and mean differences $[95 \% \mathrm{CI}]$ were calculated for domain standard scores and subdomain $v$-scale scores.

Results: Domain-level CCC ranged from $0.78[0.70,0.84]$ for Communication to 0.86 [0.76, 0.92] for Motor. Subdomain CCC ranged from 0.71 [0.62, 0.78] for Receptive Language to 0.91 $[0.85,0.95]$ for Fine Motor. Vineland-3 scores were lower than Vineland-II scores; $77 \%$ of participants had lower Adaptive Behavior Composite scores on the Vineland-3 than on the Vineland-II. For the subdomains, the magnitude of this difference depended upon the level of adaptive behavior. For Communication, the domain with the lowest $\mathrm{CCC}$, the mean difference ranged from $-13.70[-8.03,-19.35]$ for a Vineland-II score or 85 to a difference of -19.18 [-12.28, -26.87] for a Vineland-II score of 40.

Discussion: Among individuals with intellectual and developmental disabilities, the Vineland-3 produces lower scores than the Vineland-II, and these clinically significant differences tend to be larger for individuals with lower levels of ability. Thus, care must be taken in interpreting scores from the Vineland-3 relative to those obtained from the previous edition.

Keywords: Adaptive behavior, intellectual disability, developmental disorder, psychometrics, reliability 


\section{INTRODUCTION}

Adaptive behavior is used to operationalize intellectual disability (ID); diagnostic criteria require that both cognitive ability and adaptive behavior are significantly impaired during childhood or adolescence (American Psychiatric Association, 2013; World Health Organization, 2018). Because adaptive behavior refers broadly to skills in conceptual, social, and practical domains, individuals who meet diagnostic criteria, which require functional impairment, for any neurodevelopmental disability are likely to exhibit deficits in adaptive behavior. As described by Tassé and colleagues (2012), "adaptive behavior encompasses an essential dimension in a multidimensional understanding of human functioning," (p. 291) and is therefore routinely used in cross-sectional and longitudinal studies of neurodevelopmental disability.

Due to its centrality in the ID diagnostic criteria, several standardized instruments of adaptive behavior exist. Most available instruments are parent- or caregiver-interview or report [e.g., Scales of Independent Behavior, Revised (Bruininks, Woodcock, Weatherman, \& Hill, 1996), Adaptive Behavior Scale - School edition (Lambert, Nihira, \& Leland, 1993)], although some self-report instruments do exist (e.g., the Adaptive Behavior Assessment System, 3rd edition has both other and self-report forms (Harrison \& Oakland, 2015). Here, we focus on the Vineland Adaptive Behavior Scales Interview Form, which was recently published in its third edition (Sparrow, Cicchetti, \& Saulnier, 2016).

Several important changes from the Vineland-II are reflected in the Vineland-3. The overall number of items on the scale increased by $34 \%$ relative to the Vineland-II (see Supplementary Materials). Many of the new items reflect earlier/easier skills and are therefore at lower levels of ability. These new items are concentrated in a few subdomains (i.e., Receptive Communication, Written Communication, Personal Skills, and Community Skills) (Saulnier, 2016). The Vineland-3 manual also gives new instructions for assigning a score of 1 . In the Vineland-II a score of 1 was assigned when a behavior was "sometimes" or "partially" observed; in the Vineland-3, a score of 1 is assigned when the behavior is observed "sometimes." A Vineland-II rating of "partially" allowed for the possibility that prompting of the behavior may have occurred; in the Vineland-3, the behavior must be observed to occur spontaneously (although exceptions to this for a variety of items are described in the manual). The start age for some subdomains were adjusted to address the concern that some items on the Coping, Domestic, and Community domains were inappropriate for young children. Finally, while the Vineland-II included the Motor Skills domain in the computation of the Adaptive Behavior Composite for children younger than 7 years, this is not true for individuals of any age on the Vineland-3. Other more minor changes such as relocation of items between domains and updates to the language are described in the manual.

As with any new edition, it is important to consider how content, administration, and scoring differences may affect the score of an individual on the measure. This is particularly important for the Vineland, given that its use as an outcome measure in longitudinal studies and treatment trials is increasing (Bal, Kim, Cheong, \& Lord, 2015; Chatham et al., 2018; Farmer, Swineford, Swedo, \& Thurm, 2018; Farmer et al., 2019; Szatmari et al., 2015). The Vineland-3 manual does provide some information on the correspondence between the interview editions, administered to the same individual between 12 and 35 days apart (Sparrow et al., 2016). The 
mean difference (in standard deviation units) and the Pearson correlation between the VinelandII and Vineland-3 scores were calculated for samples of children and young adults grouped by age (0-2, 3-6, 7-11, 12-20 years). Sample sizes ranged from 43 to 73.

Correlations between the editions were moderate, ranging from 0.40 (Communication in the oldest subgroup) to 0.87 (Communication, Socialization, and the Adaptive Behavior Composite in the $3-6$ years subgroup). However, two scores may be strongly correlated even in the presence of a mean difference. Indeed, mean differences were nearly uniformly in the direction of lower Vineland-3 than Vineland-II scores, although Cohen's $d$ effect sizes ranged from -0.62 (Receptive Language for the Age $3-6$ group) to +0.61 (Receptive Language for the Age $12-20$ group). There was no consistent pattern across the age groups, except that the mean differences were smallest for the youngest group $(0-2$ years $)$, and mean differences were more likely to be positive (i.e., Vineland-3 scores higher than Vineland-II) for the $12-20$ age group. The manual attributes the lower Vineland-3 scores possibly to content changes, but possibly to "more lenient ...reporting" (p.222) by parents, caregivers, and teachers, although it is unclear why this would be expected in the short test-retest timeframe.

Importantly, although the level of functioning of the test-retest samples is not thoroughly described, the mean scores are very close to 100 and the sample standard deviation usually slightly less than 15, suggesting that very few participants were in the range of intellectual disability. Although a population representative sample is essential for the development of normative data, it is possible that the psychometric performance of the Vineland in such a sample is not relevant to its performance in a sample with neurodevelopmental disabilities.

For both clinical and research applications, it is necessary to understand the difference in scores yielded by the second and third editions of the Vineland in the populations for which the Vineland is most frequently used. The goal of this study was to evaluate the reliability of the Vineland-3 with the Vineland-II, with special attention to use with individuals with lower levels of ability. We hypothesized that the correlation between scores from the two tests would be very strong, but that reliability estimates would be more moderate, reflecting our hypothesis that Vineland-3 scores would be lower than Vineland-II scores on the same participants.

\section{Participants}

\section{METHODS}

Data were prospectively collected during 2017 and 2018 from a sample of convenience at two neurodevelopmental disorder research clinics. Participants at both clinics were diagnosed with a variety of neurodevelopmental disorders, including autism spectrum disorder and various neurogenetic conditions. The Neurodevelopmental and Behavioral Phenotyping Service in the Intramural Research Program of the National Institute of Mental Health (NIMH) is a research clinic. Both versions of the Vineland interview were administered to the parents/caregivers of 87 individuals enrolled in institutional review board approved research protocols sponsored by NIMH, NICHD, and NHGRI at the NIH Clinical Center. The University of California San Francisco Service, Training, Advocacy and Research (STAR) is a multidisciplinary specialty neurodevelopmental disorders clinic. Participants were clinical $(\mathrm{n}=10)$ and research referrals $(n=9)$ seen at STAR between 2017 and 2018. 


\section{Procedure}

This study was approved by the institutional review boards at both sites. Depending on age and developmental level, participants or parents/legal guardians consented to the use of these data. At both sites, the Vinelands were administered by a licensed clinical psychologist or by research assistants trained and supervised by a licensed clinical psychologist who had been trained on both the Vineland-II and Vineland-3 using materials provided by the publisher (i.e., manual, webinars) and trainings that included multiple coders for establishing initial reliability. Due to logistical constraints, it was not possible to ensure that both versions of the Vineland were administered by the same clinician. Of 106 cases, 63 were assessed by the same clinician for both Vineland versions. For this project, an effort was made to obtain both the Vineland-II and the Vineland-3 on the same day; administrations more than 7 days apart were excluded from the study. Simultaneous administrations were obtained for $n=80(91 \%)$ at NIMH and for $n=15$ (79\%) at STAR (see Table 1).

Table 1: Participant demographics $(\mathbf{N}=\mathbf{1 0 6}) . \mathrm{ABC}=$ Adaptive Behavior Composite. $\mathrm{FSIQ} / \mathrm{DQ}=$ full scale intelligence quotient or developmental quotient. The total number of raters was five at NIMH and five at STAR.

\begin{tabular}{|c|c|c|c|c|c|c|c|c|c|c|}
\hline & \multicolumn{5}{|c|}{ NIMH $(n=87)$} & \multicolumn{5}{|c|}{ STAR $(n=19)$} \\
\hline & $n$ & Mean & $S D$ & Min & $\operatorname{Max}$ & $n$ & Mean & $S D$ & Min & $\operatorname{Max}$ \\
\hline Male & $43(49 \%)$ & & & & & $14(74 \%)$ & & & & \\
\hline Age (years) & $87(100 \%)$ & 12.65 & 10.47 & 1.25 & 50.17 & $19(100 \%)$ & 16.71 & 13.01 & 1.67 & 49.58 \\
\hline Vineland-II ABC & $87(100 \%)$ & 61.43 & 20.50 & 20 & 117 & $18(95 \%)$ & 61.33 & 25.38 & 20 & 100 \\
\hline Vineland-3 $\mathrm{ABC}$ & $87(100 \%)$ & 54.70 & 22.15 & 20 & 104 & $18(95 \%)$ & 52.17 & 24.11 & 20 & 83 \\
\hline $\mathrm{FS} \mathrm{IQ} / \mathrm{DQ} \geq 85$ & $10(11 \%)$ & & & & & $7(37 \%)$ & & & & \\
\hline FS IQ/DQ $70-85$ & $10(11 \%)$ & & & & & $2(11 \%)$ & & & & \\
\hline FS IQ/DQ 55-69 & $19(22 \%)$ & & & & & 0 & & & & \\
\hline FS IQ/DQ $40-54$ & $20(23 \%)$ & & & & & 0 & & & & \\
\hline FS IQ/DQ $25-39$ & $5(6 \%)$ & & & & & 0 & & & & \\
\hline FS IQ/DQ $<25$ & $14(16 \%)$ & & & & & $2(11 \%)$ & & & & \\
\hline FS IQ/DQ missing & $9(10 \%)$ & & & & & $6(32 \%)$ & & & & \\
\hline \multicolumn{11}{|l|}{ Time between administrations } \\
\hline Same day & $82(94 \%)$ & & & & & $16(84 \%)$ & & & & \\
\hline $1-5$ days & $4(5 \%)$ & & & & & $3(16 \%)$ & & & & \\
\hline 6-7 days & $1(1 \%)$ & & & & & 0 & & & & \\
\hline $\begin{array}{l}\text { Same clinician administered both } \\
\text { forms }\end{array}$ & $60(69 \%)$ & & & & & $3(16 \%)$ & & & & \\
\hline
\end{tabular}

The comprehensive interview versions of the Vineland-II and Vineland-3 were used. Both forms yield scores for four domains (Communication, Daily Living Skills, Socialization, and Motor Skills), up to 11 subscales (three each for all domains except Motor, which has two), and an overall Adaptive Behavior Composite Score. Standard scores (population mean $=100$, $\mathrm{SD}=15$ ) are produced for the domain and composite scores, and scaled scores (called $v$-scale scores, with a population mean $=15, \mathrm{SD}=3$ ) are produced for the subscale scores. We do not report on the maladaptive behavior scales. Both forms are appropriate for use in individuals aged birth to 90 years, but some subscales are not administered to all ages. The Vineland-II has two age restrictions; one for the Written subscale ( $\geq 3$ years) and one for the Motor Skills domain $(\leq 7$ years). For the Vineland-3, the Written, Domestic, and Community subscales have a minimum age of 3, the Coping Skills subscale has a minimum age of 2, and the Motor domain has a 
maximum age of 9 years, so standard scores could not be obtained for these domains in the specified exclusionary age ranges. While both versions of the Vineland provide suggested qualitative descriptors of "high" (domain and ABC Standard Scores of 130-140), "moderately high" (domain and ABC Standard Scores of 115-129), "adequate" (domain and ABC Standard Scores of 86-114), "moderately low" (domain and ABC Standard Scores of 71-85), and "low" (domain and ABC Standard Scores of 20-70), these descriptors were not used due to insufficient precision at lower levels.

\section{Statistical Analysis}

The hypothesis in this study is about the reliability of the two versions of the Vineland, rather than their correlation, which may be high even in the presence of the hypothesized mean difference. The concordance correlation coefficient (CCC) is equivalent to the appropriately specified ICC (Shrout \& Fleiss, 1979), and is expressed in terms of the variance components of a linear mixed model (Carrasco \& Jover, 2003). Values closer to 1.0 reflect exact agreement between tests, and a threshold of 0.50 is often used as the lower bound of the "acceptable" range. Age and site were added as covariates. The ccrm package (Carrasco \& Martinez, 2015) for R version 3.5 was used to estimate the CCC and the $95 \%$ confidence interval.

To calculate the estimated mean difference between the forms, a general linear model was used to predict Vineland-3 scores from Vineland-II scores, controlling for site and age. This form of the model was selected because it allows estimation of a mean difference that might differ depending on the ability level (i.e., both a slope and an intercept are estimated). In all analyses, age was centered at 5 years to ensure interpretable parameters for all scales and subscales. To yield an interpretable intercept, the standard scores were centered at the population mean (100 for standard scores and 15 for $v$-scale scores). For the purposes of interpretation, the mean difference was estimated at relevant levels of Vineland-II score (i.e., 85, 70, 55, and 40 for standard scores, and 15, 12, 9, and 6 for $v$-scale scores). Model assumptions were evaluated via visual inspection of the residuals. All data visualization was performed in SAS/STAT 9.4. All R and SAS syntax are provided in the Supplementary Materials.

As described above, several Vineland subscales have age restrictions, which create missing data when the individual is outside the designated age range. Further, due to administration error, scores on the Written Communication subscale $(n=1)$ and the Adaptive Behavior Composite $(n=1)$ could not be computed. Listwise deletion was used per subscale; thus, the sample size for each ICC or general linear model depended on the domain/subscale: Adaptive Behavior Composite, $\mathrm{n}=105$; Written Communication, $\mathrm{n}=93$; Community, $\mathrm{n}=94$; Domestic, $n=94$; Coping, $n=101$; Motor Skills (and both subdomains), $n=44$; all other domains/subscales, $\mathrm{n}=106$.

No a priori power calculation was done, as this was a sample of convenience. However, based on published estimates (Zou, 2012), the sample size in this study is near to that required to demonstrate with $80 \%$ assurance that the confidence interval surrounding an ICC of 0.70 will exclude $0.50(\mathrm{~N}=101)$, which is often used as the lower limit of "acceptable." We adhere to the current American Statistical Association guidelines and emphasize the 95\% confidence intervals for all hypothesis tests alongside the exact p-values, and do not refer to "statistical significance." (Wasserstein, Schirm, \& Lazar, 2019). 


\section{RESULTS}

Participant demographics are described in Table 1. For each of the domains and subscales, the CCC estimates exceeded 0.70 (see Figure 1 and Table S1). There were not meaningful differences in reliability across the subscales, as evidenced by overlapping confidence intervals.

As hypothesized, Vineland-3 scores were lower than Vineland-II scores (Figure 2). At the domain level, differences between editions were largest for the Communication subscale and smallest for the Motor subscale (Table 2). In regressions predicting Vineland-3 scores, Vineland-II slopes different from 1.0 indicate that the mean difference between editions depends on the adaptive behavior level of the examinee. For example, the estimated difference for a Vineland-II Communication score of 85 was about 14 points (estimated Vineland-3 score $71.31,95 \% \mathrm{CI}=65.65-76.97)$, while the difference was about 17 for a Vineland-2 score of 55 (estimated Vineland-3 score $37.65,95 \% \mathrm{CI}=31.57-43.74$ ) (see Table 2).

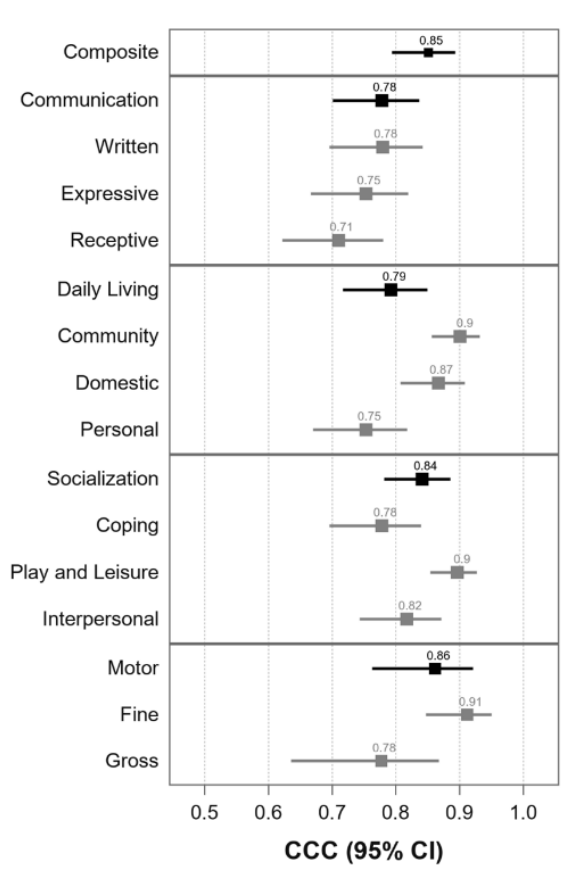

Figure 1: Agreement between editions, controlling for site and age. $\mathrm{CCC}=$ concordance correlation coefficient. $\mathrm{CCC}$ for domains (standard scores) are shaded black while subdomains ( $v$-scale scores) are shaded gray.

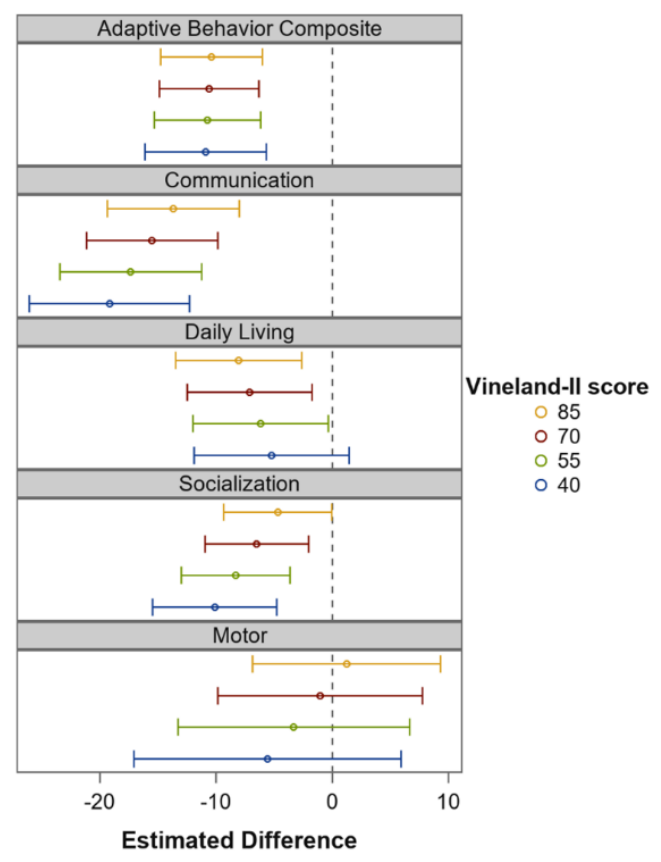

Figure 2: Estimated mean differences between Vineland-II and Vineland-3 domain scores by level of Vineland-II score, controlling for site and age. figure illustrates the estimated difference between a given Vineland-II score and the predicted Vineland-3 value, controlling for site and age (held constant at 5 years). See also Table 2 . 
Table 2: Estimated Vineland-3 scores [95\% CI] for given Vineland-II scores, controlling for age and site $(\mathrm{N}=\mathbf{1 0 6})$. Sample sizes for individual subscales/domains differ (see text). The Vineland-II score was centered at the population mean in order to yield an interpretable intercept and Vineland-3 scores were predicted for given Vineland-II scores at increments of 1 standard deviation. Site (coded as -0.5 and +0.5 ) and age (centered at 5 years, so that Motor scale scores are interpretable) were entered as covariates. The full model results are shown in Supplementary Table S2.

\begin{tabular}{lllll}
\hline Vineland-3 Scale & Vineland-II = 40 & Vineland-II = 55 & Vineland-II = 70 & Vineland-II = 85 \\
\hline Adaptive Behavior Composite & $29.10[23.89,34.31]$ & $44.26[39.68,48.84]$ & $59.42[55.14,63.7]$ & $74.58[70.21,78.96]$ \\
Communication & $20.82[13.93,27.72]$ & $37.65[31.57,43.74]$ & $54.48[48.83,60.13]$ & $71.31[65.65,76.97]$ \\
Daily Living Skills & $34.79[28.13,41.44]$ & $48.84[43.02,54.66]$ & $62.89[57.52,68.26]$ & $76.94[71.53,82.36]$ \\
Socialization & $29.88[24.55,35.21]$ & $46.69[41.99,51.38]$ & $63.5[59.05,67.94]$ & $80.31[75.66,84.95]$ \\
Motor Skills & $34.42[22.95,45.9]$ & $51.69[41.72,61.66]$ & $68.96[60.16,77.75]$ & $86.22[78.13,94.32]$ \\
& Vineland-II = 6 & Vineland-II =9 & Vineland-II = 12 & Vineland-II = 15 \\
Receptive & $3.25[1.92,4.58]$ & $6.17[4.95,7.38]$ & $9.08[7.89,10.27]$ & $12[10.72,13.28]$ \\
Expressive & $3.31[1.84,4.78]$ & $6.84[5.44,8.23]$ & $10.37[8.93,11.8]$ & $13.89[12.3,15.48]$ \\
Written Language & $2.83[1.44,4.22]$ & $6.38[5.13,7.63]$ & $9.93[8.71,11.15]$ & $13.48[12.17,14.78]$ \\
Personal & $3.25[2.15,4.35]$ & $6.3[5.29,7.32]$ & $9.35[8.31,10.4]$ & $12.41[11.24,13.58]$ \\
Domestic & $4.36[3.23,5.48]$ & $7.16[6.16,8.16]$ & $9.96[9.01,10.91]$ & $12.77[11.78,13.76]$ \\
Community & $5.71[4.81,6.6]$ & $8.68[7.85,9.51]$ & $11.65[10.83,12.47]$ & $14.63[13.76,15.5]$ \\
Interpersonal Relationships & $3.96[2.96,4.96]$ & $7.34[6.4,8.27]$ & $10.72[9.75,11.68]$ & $14.09[13.02,15.17]$ \\
Play and Leisure & $5.12[4.32,5.91]$ & $8.49[7.73,9.25]$ & $11.86[11.07,12.65]$ & $15.23[14.34,16.12]$ \\
Coping Skills & $5.06[3.98,6.15]$ & $8.01[7.01,9]$ & $10.95[9.92,11.98]$ & $13.89[12.72,15.07]$ \\
Gross Motor & $2.33[0.11,4.55]$ & $6.75[4.89,8.61]$ & $11.17[9.57,12.77]$ & $15.59[14.09,17.08]$ \\
Fine Motor & $5.49[3.92,7.05]$ & $8.71[7.31,10.1]$ & $11.93[10.63,13.23]$ & $15.15[13.84,16.46]$ \\
\hline & & &
\end{tabular}

To assist in interpretation of these discrepancies at the individual level, the axes of Figure 3 are categorized into Average or above $(\geq 85)$, Borderline $(70-84)$, Mild $(55-69)$, Moderate $(40-54)$, Severe $(25-39)$, Profound $(20-24)$ impairment levels. For the Adaptive Behavior Composite, six participants were classified by the Vineland-3 two levels lower than by the Vineland-II (e.g., Severe versus Mild).

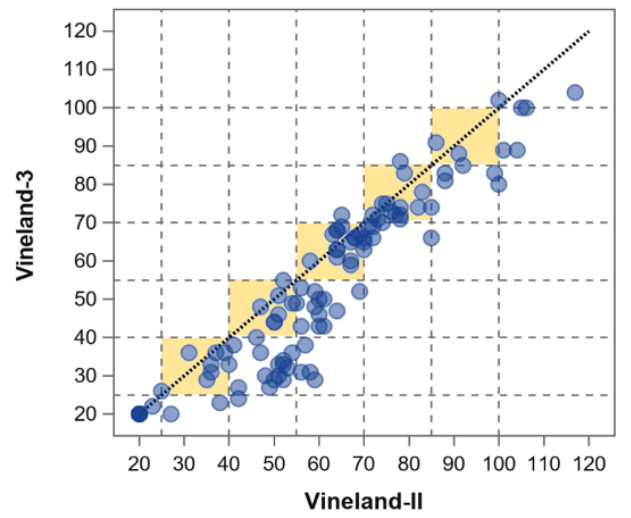

Figure 3: Raw Adaptive Behavior Composite data. Dotted lines are for reference and demarcate commonly used levels of intellectual disability $(<25=$ Profound, $25-39=$ Severe, $40-54=$ Moderate, $55-74=$ Mild, 75-84 = Borderline, $\geq 85=$ Average) . The diagonal dotted reference line reflects a perfect 1:1 relationship; individuals above this diagonal line had higher Vineland-3 scores, while individuals below the line had higher Vineland-II scores. Shaded areas of graph capture individuals falling within the same group classification (e.g., mild or moderate ID); individuals whose classifications shifted fall outside of the shaded areas. See Table S2 for intercepts and slopes of the Adaptive Behavior Composite and all other subscales. 


\section{DISCUSSION}

This is the first independent study of the reliability between the Vineland-II and Vineland-3. While moderate reliability was observed, the Vineland-3 produces lower scores than the Vineland-II across all domains and ability levels. These findings are consistent with those published in the Vineland-3 manual, but they extend that work by evaluating the scales in a sample of individuals with various neurodevelopmental disabilities, rather than the populationbased samples used in the manual. Thus, these psychometric data are more readily generalizable to the types of samples in which the Vineland is often used.

Even scores with a large mean difference may exhibit a strong correlation; for this reason it is important to characterize reliability using an agreement statistic rather than a Pearson correlation (Cicchetti, 2017). In this study, we chose the concordance correlation coefficient (CCC), which in this case is identical to the intraclass correlation calculated for absolute agreement. The lower boundaries of the CCC confidence intervals were generally around 0.70 ; while this is acceptable, it reflects the fact that scores from the two instruments do differ. Specifically, we found that the Vineland-3 produces scores that are lower than the Vineland-II, and this difference is larger at lower levels of adaptive behavior. In some cases, the estimated mean difference in scores exceeded 15 points. Differences of this magnitude resulted in a clinically significant shift of ID classification to two levels below their Vineland-II classification.

The mean difference in scores between the Vineland-II and the Vineland-3 has important implications for clinical and research practice. Clinically, the Vineland is used to monitor an individual's progress or to establish or confirm ongoing eligibility for support. Our results indicate that when the clinician adopts the Vineland-3, the adaptive behavior estimates for a given client will be lower than they would have been with the Vineland-II. This decrease must be interpreted with caution, as it likely reflects a change in measure rather than a change in adaptive behavior functioning. Similar caution is needed in research settings, where findings with the Vineland-3 will be compared to existing work using the Vineland-II. The difference in Vineland3 and Vineland-II scores is especially relevant to treatment studies, as a change in measure could be mistaken for a treatment effect. Thus, these results provide a strong argument against transitioning between editions mid-study; should this occur, researchers must account for the form version in any statistical analysis. For the Adaptive Behavior Composite, the mean differences were relatively constant across levels of adaptive behavior, so a main effect of form could sufficiently account for the edition change. However, particularly for the Communication domain, the mean difference in editions varied by level of function level. In this case, a simple solution is not evident.

\section{Limitations}

This was a sample of convenience, and we were therefore unable to adequately evaluate potential moderators of the reliability between forms, such as age. For example, multiple reports suggest that Vineland-II scores tend to decline with age in several neurodevelopmental disorders (Ghezzo et al., 2014; Kanne et al., 2011; Klaiman et al., 2014). This effect of age may moderate the reliability between Vineland-II and Vineland-3. Because of the relatively small sample and inadequate representation at all combinations of age and adaptive behavior, we were unable to 
properly explore this possibility. Future work, especially that by the test developers, should address this possibility.

Other limitations stem from the fact that this was a sample of convenience, including that age restrictions on the Motor domain meant that those analyses were underpowered, and that the results of this study may not be generalizable to all clinical and research settings. Because the mean difference between editions depended upon adaptive behavior level for some subscales, the composition of the sample directly affected the mean differences we obtained. To mitigate this fact, we estimated the mean differences at several reference levels. We did not randomly assign either the order in which the Vineland-II and Vineland-3 were presented or the clinician, and therefore cannot statistically account for these effects. The short timeframe between administrations, which were frequently simultaneous, makes this a true test of reliability, untainted by true changes in behavior. In many cases, these were not truly independent administrations; for this reason, our estimates of reliability should be viewed as an upper limit on true reliability.

Finally, although reliability was the focus of this study, a question of validity arises. Which is a truer reflection of the adaptive function of the individual, the higher Vineland-II score or the lower Vineland-3 score? Unfortunately, there is no external gold standard for evaluation of adaptive behavior, so we are unable to judge the editions' respective validity.

\section{Conclusion}

The recently published Vineland-3 Adaptive Behavior Scales (Comprehensive Interview Form) contains many changes, including new items and different scoring instructions. As with any standardized measure, it is important for both researchers and clinicians to understand the correspondence between the new and previous editions. In this study, we generalize the findings published in the Vineland-3 manual, which documented slightly lower Vineland-3 scores among typically developing individuals. Among individuals with intellectual and developmental disabilities, the Vineland-3 produces lower scores than the Vineland-II, and these clinically significant differences tend to be larger for individuals with lower levels of adaptive behavior. Thus, care must be taken in interpreting scores from the Vineland-3 relative to those obtained from the previous edition. 


\section{REFERENCES}

American Psychiatric Association. (2013). Diagnostic and statistical manual of mental disorders : DSM-5 (5th ed.). Washington, D.C.: American Psychiatric Association.

Bal, V. H., Kim, S.-H., Cheong, D., \& Lord, C. (2015). Daily living skills in individuals with autism spectrum disorder from 2 to 21 years of age. Autism, 19(7), 774-784.

Bruininks, R. H., Woodcock, R. W. B. K., Weatherman, R. F., \& Hill, B. K. (1996). Scales of Independent Behavior-Revised. SIB-R: Riverside Publishing Company Itasca, IL.

Carrasco, J. L., \& Jover, L. (2003). Estimating the generalized concordance correlation coefficient through variance components. Biometrics, 59(4), 849-858.

Carrasco, J. L., \& Martinez, J. P. (2015). Concordance Correlation Coefficient for Repeated (and Non-Repeated) Measures. In: R package version 1.2.1.

Chatham, C., Taylor, K., Charman, T., Liogier D'Ardhuy, X., Eule, E., Fedele, A., ... del Valle Rubido, M. (2018). Adaptive behavior in autism: Minimal clinically important differences on the Vineland-II. Autism Research, 11(2), 270-283.

Cicchetti, D. (2017). Choice of agreement statistics: A discussion of the underlying biostatistics, with heuristic examples from the Vineland-3. Retrieved from https://images.pearsonclinical.com/images/assets/vineland-3/Vineland3 BiostatisticsAgreement 1A.pdf

Farmer, C., Swineford, L., Swedo, S. E., \& Thurm, A. (2018). Classifying and characterizing the development of adaptive behavior in a naturalistic longitudinal study of young children with autism. Journal of Neurodevelopmental Disorders, 10(1), 1.

Farmer, C., Thurm, A., Farhat, N., Bianconi, S., Keener, L. A., \& Porter, F. D. (2019). LongTerm Neuropsychological Outcomes from an Open-Label Phase I/IIa Trial of 2Hydroxypropyl- $\beta$-Cyclodextrins (VTS-270) in Niemann-Pick Disease, Type C1. CNS drugs, $1-7$.

Ghezzo, A., Salvioli, S., Solimando, M. C., Palmieri, A., Chiostergi, C., Scurti, M., . . Follo, D. (2014). Age-related changes of adaptive and neuropsychological features in persons with Down Syndrome. PLoS One, 9(11), e113111.

Harrison, P. L., \& Oakland, T. (2015). ABAS-3: Adaptive behavior assessment system: Western Psychological Services Los Angeles, CA.

Kanne, S. M., Gerber, A. J., Quirmbach, L. M., Sparrow, S. S., Cicchetti, D. V., \& Saulnier, C. A. (2011). The Role of Adaptive Behavior in Autism Spectrum Disorders: Implications for Functional Outcome. Journal of autism and developmental disorders, 41(8), 10071018. Retrieved from $<$ Go to ISI $>$ ://WOS:000293977500003. doi:10.1007/s10803-010$1126-4$

Klaiman, C., Quintin, E. M., Jo, B., Lightbody, A. A., Hazlett, H. C., Piven, J., . . Reiss, A. L. (2014). Longitudinal profiles of adaptive behavior in fragile $\mathrm{X}$ syndrome. Pediatrics, 134(2), 315-324. doi:10.1542/peds.2013-3990

Lambert, N., Nihira, K., \& Leland, H. (1993). ABS-S: 2: AAMR Adaptive Behavior Scale: School: Pro-ed.

Saulnier, C. (2016). Vineland Adaptive Behavior Scales, Third Edition [Slides].

Shrout, P. E., \& Fleiss, J. L. (1979). Intraclass correlations: uses in assessing rater reliability. Psychological Bulletin, 86(2), 420.

Sparrow, S. S., Cicchetti, D. V., \& Saulnier, C. A. (2016). Vineland Adaptive Behavior Scales, Third Edition. San Antonio, TX: Pearson. 
Szatmari, P., Georgiades, S., Duku, E., Bennett, T. A., Bryson, S., Fombonne, E., . . . Vaillancourt, T. (2015). Developmental trajectories of symptom severity and adaptive functioning in an inception cohort of preschool children with autism spectrum disorder. JAMA psychiatry, 72(3), 276-283.

Tassé, M. J., Schalock, R. L., Balboni, G., Bersani Jr, H., Borthwick-Duffy, S. A., Spreat, S., . . . Zhang, D. (2012). The construct of adaptive behavior: Its conceptualization, measurement, and use in the field of intellectual disability. Am J Intellect Dev Disabil, 117(4), 291-303.

Wasserstein, R. L., Schirm, A. L., \& Lazar, N. A. (2019). Moving to a World Beyond "p <0.05". The American Statistician, 73(sup1), 1-19. Retrieved from https://doi.org/10.1080/00031305.2019.1583913. doi:10.1080/00031305.2019.1583913

World Health Organization. (2018). International statistical classification of diseases and related health problems (11th Revision). Retrieved from https://icd.who.int/browse11/1m/en

Zou, G. (2012). Sample size formulas for estimating intraclass correlation coefficients with precision and assurance. Statistics in Medicine, 31(29), 3972-3981. 


\section{SUPPORTING INFORMATION}

TITLE: Reliability of the Vineland Adaptive Behavior Scales, Third Edition CONTENTS:

1. Syntax for concordance correlation coefficient analysis

2. Syntax for mean difference analysis

3. Table S0: Number of items on each Vineland edition, by subscale

4. Table S1: Concordance correlation coefficients estimated by variance components analysis, controlling for age and site

5. Table S2: Parameter estimates from general linear model predicting Vineland-3 score as a function of Vineland-II score by age

Syntax for CCC analysis (R Version 3.5)

!Create function to extract CCC value, with 95\% CI, for each variable pair !Where USE is a dataset that contains one row per subject ID per VERSION per SUBSCALE, controlling for AGECTR5 and SITE.

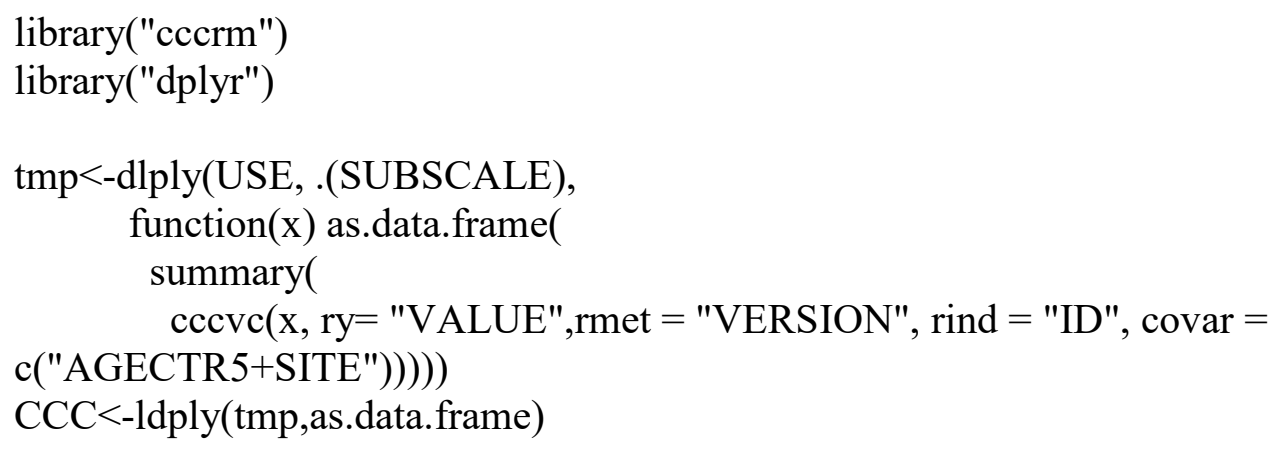

Syntax for mean difference analysis

*Where REG is a dataset that contains one row per subject, with one variable for each Vineland form score, _2ctr is the Vineland-II score centered at the population mean (100 for standard scores, 10 for V-scale scores), 3 is the Vineland-3 score (not centered), site is the site ID ( -0.5 for $\mathrm{NIH}$ and +0.5 for STAR), agectr 5 is age in years centered at 5 , and type indicates whether the subscale is a standard score (SS) or V-scale score (V). Estimate statements are used to compute the predicted_3 value at a given _2 value and a given age.

proc mixed data $=\mathrm{REG}$;

BY subscale type;

where TYPE = 'SS';

model_3 = site agectr5_2ctr/ s cl;

estimate 'VABS-3 at VABS-2 = 40, age $=5$ ' intercept 1 site $\mathbf{0 . 5} \_2 \mathrm{ctr}-\mathbf{6 0}$, 
'VABS-3 at VABS-2 $=55$, age $=5$ ' intercept 1 site $\mathbf{0 . 5} \_$ctr $-\mathbf{4 5}$, 'VABS-3 at VABS-2 $=70$, age $=5$ ' intercept 1 site $\mathbf{0 . 5} \_$ctr $\mathbf{- 3 0}$, 'VABS-3 at VABS-2 $=85$, age $=5$ ' intercept 1 site $0.5 \_2 \mathrm{ctr}-15 / \mathrm{cl}$; run;

proc mixed data $=\mathrm{REG}$;

BY SUBSCALE TYPE;

where TYPE = 'V';

model_3 = site agectr $5 \mid 2 \mathrm{ctr} / \mathrm{s}$ cl;

estimate 'VABS-3 at VABS-2 $=6$, age $=5$ ' intercept 1 site $\mathbf{0 . 5}$ 2 ctr $\mathbf{- 9}$, 'VABS-3 at VABS-2 $=9$, age $=5$ ' intercept 1 site $0.5 \_2$ ctr -6 , 'VABS-3 at VABS-2 $=12$, age $=5$ ' intercept 1 site $\mathbf{0 . 5} \_$ctr $\mathbf{- 3}$, 'VABS-3 at VABS-2 $=15$, age $=5$ ' intercept 1 site $0.5 / \mathrm{cl}$;

run; 
Table S0. Number of items on each form

\begin{tabular}{|c|c|c|}
\hline & Vineland-3 & Vineland-II \\
\hline Communication & 126 & 99 \\
\hline Receptive & 39 & 20 \\
\hline Expressive & 49 & 54 \\
\hline Written & 38 & 25 \\
\hline Daily Living Skills & 143 & 109 \\
\hline Personal & 55 & 41 \\
\hline Domestic & 30 & 24 \\
\hline Community & 58 & 44 \\
\hline Socialization & 169 & 99 \\
\hline Interpersonal Relationships & 43 & 38 \\
\hline Play and Leisure & 36 & 31 \\
\hline Coping Skills & 33 & 30 \\
\hline Motor Skills & 77 & 76 \\
\hline Gross Motor & 43 & 40 \\
\hline Fine Motor & 34 & 36 \\
\hline
\end{tabular}


Table S1. Concordance correlation coefficients estimated by variance components analysis, controlling for age and site

\begin{tabular}{lllll}
\hline Domain & Subscale & CCC & $\begin{array}{l}\text { CCC } \\
\mathbf{9 5 \%} \\
\text { CI } \\
\text { lower } \\
\text { bound }\end{array}$ & $\begin{array}{l}\text { CCC } \\
\mathbf{9 5 \%} \\
\text { CI } \\
\text { upper } \\
\text { bound }\end{array}$ \\
\hline $\begin{array}{l}\text { Adaptive Behavior } \\
\text { Composite }\end{array}$ & $\begin{array}{l}\text { Adaptive Behavior } \\
\text { Composite }\end{array}$ & 0.85078 & 0.79396 & 0.89287 \\
& Receptive & 0.70983 & 0.62185 & 0.78011 \\
& Expressive & 0.75291 & 0.66621 & 0.81953 \\
& Written & 0.77911 & 0.69572 & 0.84179 \\
Daily Living Skills & Communication & 0.77768 & 0.7013 & 0.8364 \\
& Personal & 0.75311 & 0.67015 & 0.81748 \\
& Domestic & 0.86634 & 0.80689 & 0.90842 \\
Socialization & Community & 0.90022 & 0.85629 & 0.93122 \\
& Daily Living Skills & 0.79236 & 0.71681 & 0.84952 \\
& Interpersonal & 0.81702 & 0.74301 & 0.87129 \\
& $\begin{array}{l}\text { Relationships } \\
\text { Play and Leisure }\end{array}$ & 0.89627 & 0.85394 & 0.92682 \\
& Coping Skills & 0.77794 & 0.69608 & 0.83983 \\
& Socialization & 0.84133 & 0.78174 & 0.88569 \\
Motor Skills & Gross Motor & 0.77675 & 0.63552 & 0.86769 \\
& Fine Motor & 0.91213 & 0.84707 & 0.95025 \\
& Motor Skills & 0.8617 & 0.76286 & 0.92119 \\
\hline & & &
\end{tabular}


Table S2. Parameter estimates from general linear model predicting Vineland-3 score as a function of Vineland-II score by age

\begin{tabular}{|c|c|c|c|c|c|c|c|c|}
\hline $\begin{array}{l}\text { Vineland-3 } \\
\text { Outcome }\end{array}$ & Effect & Estimate & $\begin{array}{c}\text { Standard } \\
\text { Error }\end{array}$ & Lower & Upper & $\mathbf{t}$ & DF & $\mathbf{p}$ \\
\hline \multirow{4}{*}{$\begin{array}{l}\text { Adaptive Behavior } \\
\text { Composite }\end{array}$} & Intercept & 91.25 & 1.82 & 87.64 & 94.85 & 50.19 & 101.00 & $<.0001$ \\
\hline & Site & -3.01 & 2.18 & -7.32 & 1.31 & -1.38 & 101.00 & 0.17 \\
\hline & Age & 0.12 & 0.09 & -0.05 & 0.30 & 1.41 & 101.00 & 0.16 \\
\hline & Vineland-II score & 1.01 & 0.04 & 0.92 & 1.10 & 22.65 & 101.00 & $<.0001$ \\
\hline \multirow[t]{4}{*}{ Communication } & Intercept & 89.15 & 2.22 & 84.74 & 93.56 & 40.13 & 102.00 & $<.0001$ \\
\hline & Site & -2.03 & 2.88 & -7.74 & 3.67 & -0.71 & 102.00 & 0.48 \\
\hline & Age & 0.46 & 0.12 & 0.22 & 0.70 & 3.80 & 102.00 & 0.00 \\
\hline & Vineland-II score & 1.12 & 0.05 & 1.01 & 1.23 & 20.46 & 102.00 & $<.0001$ \\
\hline \multirow{4}{*}{ Daily Living Skills } & Intercept & 90.96 & 2.25 & 86.50 & 95.42 & 40.42 & 102.00 & $<.0001$ \\
\hline & Site & 0.07 & 2.76 & -5.41 & 5.55 & 0.02 & 102.00 & 0.98 \\
\hline & Age & -0.17 & 0.11 & -0.38 & 0.05 & -1.52 & 102.00 & 0.13 \\
\hline & Vineland-II score & 0.94 & 0.06 & 0.83 & 1.05 & 16.90 & 102.00 & $<.0001$ \\
\hline \multirow[t]{4}{*}{ Motor } & Intercept & 101.14 & 2.57 & 95.96 & 106.33 & 39.42 & 40.00 & $<.0001$ \\
\hline & Site & 4.70 & 4.73 & -4.85 & 14.25 & 0.99 & 40.00 & 0.33 \\
\hline & Age & 2.89 & 0.90 & 1.07 & 4.71 & 3.20 & 40.00 & 0.00 \\
\hline & Vineland-II score & 1.15 & 0.07 & 1.00 & 1.30 & 15.48 & 40.00 & $<.0001$ \\
\hline \multirow[t]{4}{*}{ Socialization } & Intercept & 96.03 & 1.88 & 92.30 & 99.77 & 51.00 & 102.00 & $<.0001$ \\
\hline & Site & 2.18 & 2.28 & -2.35 & 6.70 & 0.95 & 102.00 & 0.34 \\
\hline & Age & 0.11 & 0.09 & -0.07 & 0.29 & 1.25 & 102.00 & 0.21 \\
\hline & Vineland-II score & 1.12 & 0.05 & 1.03 & 1.22 & 23.34 & 102.00 & $<.0001$ \\
\hline \multirow[t]{4}{*}{ Receptive } & Intercept & 12.12 & 0.45 & 11.23 & 13.01 & 27.16 & 102.00 & $<.0001$ \\
\hline & Site & -0.24 & 0.61 & -1.45 & 0.96 & -0.40 & 102.00 & 0.69 \\
\hline & Age & 0.01 & 0.02 & -0.04 & 0.05 & 0.24 & 102.00 & 0.81 \\
\hline & Vineland-II score & 0.97 & 0.06 & 0.85 & 1.09 & 16.30 & 102.00 & $<.0001$ \\
\hline \multirow[t]{2}{*}{ Expressive } & Intercept & 13.80 & 0.58 & 12.65 & 14.96 & 23.71 & 102.00 & $<.0001$ \\
\hline & Site & 0.18 & 0.71 & -1.23 & 1.59 & 0.25 & 102.00 & 0.80 \\
\hline
\end{tabular}




\begin{tabular}{|c|c|c|c|c|c|c|c|c|}
\hline \multirow{5}{*}{$\begin{array}{l}\text { Written } \\
\text { Communication }\end{array}$} & Age & 0.04 & 0.03 & -0.01 & 0.09 & 1.66 & 102.00 & 0.10 \\
\hline & Vineland-II score & 1.18 & 0.07 & 1.04 & 1.31 & 17.11 & 102.00 & $<.0001$ \\
\hline & Intercept & 13.48 & 0.46 & 12.55 & 14.40 & 29.05 & 89.00 & $<.0001$ \\
\hline & Site & 0.00 & 0.61 & -1.20 & 1.21 & 0.01 & 89.00 & 0.99 \\
\hline & Age & 0.07 & 0.02 & 0.02 & 0.11 & 2.69 & 89.00 & 0.01 \\
\hline \multirow{3}{*}{ Personal } & Vineland-II score & 1.18 & 0.06 & 1.06 & 1.31 & 18.79 & 89.00 & $<.0001$ \\
\hline & Intercept & 12.60 & 0.46 & 11.69 & 13.52 & 27.39 & 102.00 & $<.0001$ \\
\hline & Site & -0.39 & 0.52 & -1.43 & 0.65 & -0.74 & 102.00 & 0.46 \\
\hline \multirow{5}{*}{ Domestic } & Age & 0.06 & 0.02 & 0.02 & 0.10 & 3.12 & 102.00 & 0.00 \\
\hline & Vineland-II score & 1.02 & 0.06 & 0.90 & 1.13 & 17.68 & 102.00 & $<.0001$ \\
\hline & Intercept & 13.36 & 0.35 & 12.66 & 14.06 & 37.92 & 90.00 & $<.0001$ \\
\hline & Site & -1.19 & 0.48 & -2.14 & -0.24 & -2.48 & 90.00 & 0.02 \\
\hline & Age & 0.00 & 0.02 & -0.04 & 0.04 & 0.18 & 90.00 & 0.86 \\
\hline \multirow{3}{*}{ Community } & Vineland-II score & 0.93 & 0.05 & 0.84 & 1.03 & 18.82 & 90.00 & $<.0001$ \\
\hline & Intercept & 14.01 & 0.32 & 13.38 & 14.64 & 44.24 & 90.00 & $<.0001$ \\
\hline & Site & 1.24 & 0.41 & 0.43 & 2.05 & 3.03 & 90.00 & 0.00 \\
\hline \multirow{5}{*}{$\begin{array}{l}\text { Interpersonal } \\
\text { Relationships }\end{array}$} & Age & 0.02 & 0.02 & -0.01 & 0.05 & 1.23 & 90.00 & 0.22 \\
\hline & Vineland-II score & 0.99 & 0.04 & 0.92 & 1.07 & 26.18 & 90.00 & $<.0001$ \\
\hline & Intercept & 13.88 & 0.38 & 13.12 & 14.64 & 36.14 & 102.00 & $<.0001$ \\
\hline & Site & 0.43 & 0.48 & -0.52 & 1.38 & 0.90 & 102.00 & 0.37 \\
\hline & Age & 0.16 & 0.02 & 0.13 & 0.20 & 8.99 & 102.00 & $<.0001$ \\
\hline \multirow{3}{*}{ Play and Leisure } & Vineland-II score & 1.13 & 0.05 & 1.03 & 1.22 & 22.68 & 102.00 & $<.0001$ \\
\hline & Intercept & 14.79 & 0.32 & 14.16 & 15.42 & 46.66 & 102.00 & $<.0001$ \\
\hline & Site & 0.88 & 0.39 & 0.10 & 1.65 & 2.24 & 102.00 & 0.03 \\
\hline \multirow{5}{*}{ Coping Skills } & Age & 0.01 & 0.01 & -0.02 & 0.04 & 0.85 & 102.00 & 0.40 \\
\hline & Vineland-II score & 1.12 & 0.04 & 1.05 & 1.20 & 28.90 & 102.00 & $<.0001$ \\
\hline & Intercept & 13.86 & 0.41 & 13.04 & 14.68 & 33.54 & 97.00 & $<.0001$ \\
\hline & Site & 0.06 & 0.50 & -0.94 & 1.06 & 0.12 & 97.00 & 0.90 \\
\hline & Age & -0.04 & 0.02 & -0.07 & 0.00 & -2.04 & 97.00 & 0.04 \\
\hline
\end{tabular}




\begin{tabular}{llccccccc}
\hline & Vineland-II score & 0.98 & 0.06 & 0.86 & 1.10 & 16.30 & 97.00 & $<.0001$ \\
Gross Motor & Intercept & 15.68 & 0.47 & 14.72 & 16.64 & 33.03 & 40.00 & $<.0001$ \\
& Site & -0.19 & 0.96 & -2.13 & 1.75 & -0.20 & 40.00 & 0.84 \\
& Age & 0.45 & 0.17 & 0.11 & 0.79 & 2.67 & 40.00 & 0.01 \\
& Vineland-II score & 1.47 & 0.09 & 1.29 & 1.65 & 16.73 & 40.00 & $<.0001$ \\
\multirow{3}{*}{ Fine Motor } & Intercept & 14.66 & 0.41 & 13.84 & 15.48 & 36.18 & 40.00 & $<.0001$ \\
& Site & 0.98 & 0.72 & -0.48 & 2.45 & 1.36 & 40.00 & 0.18 \\
& Age & 0.10 & 0.15 & -0.19 & 0.40 & 0.71 & 40.00 & 0.48 \\
& Vineland-II score & 1.07 & 0.06 & 0.95 & 1.19 & 17.95 & 40.00 & $<.0001$ \\
\hline
\end{tabular}

Note: Age was centered at 5 years. Vineland-II score was centered at the population mean, which was 15 for V-scale scores and 100 for standard scores. Vineland-3 scores were not centered. Thus the intercept of each model is the estimated Vineland-3 score at the Vineland-II population mean. Site was coded as -0.5 for NIMH and +0.5 for STAR. 